\title{
Density Functional Theory of Laser-Induced Freezing in Colloidal Suspensions
}

\author{
J. Chakrabarti, H. R. Krishnamurthy, * and A. K. Sood* \\ Department of Physics, Indian Institute of Science, Bangalore 560 012, India
}

\begin{abstract}
We reexamine the density functional theory of laser-induced freezing, i.e., the freezing of a colloidal liquid into crystalline order in the presence of an external periodic modulating potential $V_{e}$ with its wave vectors tuned to the ordering wave vector in the liquid phase. We show definitively that the initial first order freezing transition (at small $V_{e}$ ) changes to a continuous one (at large $V_{e}$ ) via a tricritical point provided the modulation wave vectors are suitably chosen. We also present predictions for the parameter values of the tricritical points for a realistic colloidal system.
\end{abstract}

Several years ago Chowdhury, Ackerson, and Clark [1] demonstrated the very interesting phenomenon of "laser induced freezing" in a 2D suspension of strongly interacting colloidal particles. Here the colloidal particles were subject to a $1 \mathrm{D}$ periodic modulating potential $V_{e}$ induced (via their polarizabilities) by a standing wave pattern of interfering laser fields. The wave vector of the modulation was tuned to be at the first peak in the direct correlation function (DCF) of the colloidal liquid in the absence of $V_{e}$. Using light scattering experiments, Chowdhury and co-workers [1] then showed that the colloidal system responds to $V_{e}$ by forming a 2D (modulated) crystalline phase, with predominantly hexagonal order similar to that of the colloidal crystal obtained in the absence of $\mathrm{V}$,

Chowdhury and co-workers [1] also analyzed this phenomenon theoretically in terms of a simple LandauAlexander-McTague [2] theory. Using this, they concluded that the transition from the 1D modulated liquid phase (i.e., the liquid phase with weak modulation induced by $V_{e}$ ) to the 2D (modulated) crystalline phase can be made continuous for sufficiently large laser fields. This, if true, would make for a fascinating critical phenomenon. However, while later studies using direct macroscopic observations [3] and Monte Carlo simulations [4] have confirmed the existence of laser induced freezing, their conclusions regarding the nature of the transition between the modulated liquid and the crystal have not been definitive.

In the past two decades the density functional theory (DFT) of freezing pioneered by Ramakrishnan and Yussouff [5] has been used extensively [6] to study liquidsolid transitions. The DFT, unlike the phenomenological Landau theories, is capable of predicting [7] the phase diagrams of experimental colloidal systems [8] without adjustable parameters. It is of obvious interest to ask what DFT has to say regarding the phenomenon of laser induced freezing. The only work that we know of in this direction is that of $\mathrm{Xu}$ and Baus [9]. They studied the DFT of a 3D hard sphere system with its DCF being modeled by the Percus-Yevick DCF, in the presence of a 3D simple-cubic modulating potential $V_{e}$ commensurate with the fcc lattice to which the system freezes in the absence of $\mathrm{V}$, However, their results seemed to indicate that the fluid to crystal transition remains first order no matter how large $V_{e}$ is, in contradiction to the results of Ref. [1].

In this paper we reexamine the DFT for laser induced freezing, i.e., for a model colloidal system subject to an external periodic modulating potential $V_{e}$ with a lower symmetry than that of the crystalline phase of that colloid. We show definitively that, with increasing $V$,, the transition from the modulated liquid to the modulated crystalline phase changes from jrst order to continuous via a tricritical point (TCP)provided certain conditions, stated below, are satisjed by the wave vectors of the modulating potential. We reestablish the conclusions of [1], but liberate them from the limitations of the Landau theory $[1,2]$ (which, as we show, has no stable crystalline phase for large $V_{e}$ ). Furthermore, we apply our theory to real colloidal systems and predict the parameter ranges for which they exhibit such continuous liquid crystal transitions.

In order to understand the qualitative features of our DFT of real colloids, it is useful to consider first the simplest version of DFT, for an incompressible 2D liquid, which when $V_{e}=0$ freezes into a triangular lattice $[1,10]$ with its six smallest reciprocal lattice vectors $(\mathrm{RLV})\left\{\vec{g}_{i}^{(o)}\right\}=(0, \pm 1) q_{0},( \pm \sqrt{3} / 2, \pm 1 / 2) q_{0}$. Here $q_{0}$, as is known from DFT, corresponds to the first peak of the liquid DCF $c^{(2)}(q)$. We take $V_{e}$ to be 1D modulated, with wave vectors $\left\{\overrightarrow{\boldsymbol{g}}_{i}^{(f)}\right\}=(\boldsymbol{0}, \pm 1) q_{0}$ from this set $\left\{\vec{g}_{i}^{(o)}\right\}$. The order parameters in the simplest DFT are the Fourier components, with wave vectors $\left\{\overrightarrow{\boldsymbol{g}}_{i}^{(o)}\right\}$, of the molecular field $\xi(r) \equiv \ln \left[\rho(r) / \rho_{0}\right]$, where $\rho(r)$ is the local density in the modulated liquid or crystal $[5,6,11]$ and $\rho_{0}$ is the density of the liquid. From symmetry considerations it follows that for the crystal with $V_{e}$, the order parameters for the $\operatorname{RLV}$ set $\left\{\overrightarrow{\boldsymbol{g}}_{i}^{(f)}\right\}$ will have one value, say, $\boldsymbol{\xi}_{f}$, and those for the rest of the RLV's in the smallest set $\left\{\vec{g}_{i}^{(d)}\right\}\left[\equiv\left\{\vec{g}_{i}^{(o)}\right\}-\left\{\vec{g}_{i}^{(f)}\right\}\right]$, another value, say, $\boldsymbol{\xi}_{d}$. Let $c_{1}^{(2)} \equiv$ $\rho_{0} c^{(2)}\left(q_{0}\right)$. According to density functional theory, the excess free-energy cost (relative to the liquid) of creating 
these order parameters is given (in the incompressible limit) by $[5,6,11]$

$$
\beta F=-\ln \Phi \stackrel{n_{f} \xi_{f}^{L}+n_{d} \xi_{d}^{2}}{\sim} \beta V_{e} .
$$

Here $\Phi \equiv v_{0}^{-1} \int^{\mathrm{cell}} d^{d} \vec{r} \exp \left[\xi_{f} \phi_{f}(\vec{r})+\xi_{d} \phi_{d}(\vec{r})\right]$, where $\phi_{f}(\vec{r}) \equiv \sum_{j} \exp \left(i \vec{g}_{j}^{(f)} \cdot \vec{r}\right)$ and $\phi_{d}(\vec{r}) \equiv \sum_{j} \exp \left(i \vec{g}_{j}^{(d)} \cdot \vec{r}\right)$, $n_{f}$ and $n_{d}$ are the number of wave vectors in the set $\left\{\vec{g}_{i}^{(f)}\right\}$ and $\left\{\vec{g}_{i}^{(d)}\right\}$, respectively, and the integration is over a unit cell of volume $v_{0}$ of the (modulated) crystal. This free energy [12] is to be minimized in the space of the order parameters for a given $-\beta V_{e}$ and $c_{1}^{(2)}$ to find the phase diagram. We do this by solving numerically the two self-consistency equations obtainable from (1) by setting $\frac{\partial F}{\partial \xi_{f}}=\frac{\partial F}{\partial \xi_{d}}=0$. In this way we obtain the phase diagram of Fig. 1, which has two phases: (1) The modulated liquid with $\xi_{f} \neq 0$ but $\xi_{d}=0$; this goes continuously to the ordinary liquid phase with $\xi_{f}=\xi_{d}=0$ as $V_{e} 0$. (2) The (modulated) crystalline phase with $\xi_{f} \neq \xi_{d} \neq 0$; as $V_{e} \quad 0$, this goes into the ordinary crystalline phase with $\xi_{\perp}=\xi_{d} \# 0$. As is known [10], at $V_{e}=0$, the liquid crystal transition is first order and takes place at $c_{1}^{(2)}=0.857$ (which corresponds to the first peak height of the liquid structure factor $S_{\max }=7.14$ ). When $V_{e}$ is turned on, the transition remains first order, but moves to smaller values of $c_{1}^{(2)}$, as indicated by the solid line in Fig. 1. Thus $V_{e}$ facilitates the liquid-crystal transition, which is the phenomenon of laser induced freezing. This transition is characterized by a discontinuous change in $\xi_{f}$ as well as by a discontinuous development of $\xi_{d}$. However, the jumps in $\xi_{d}$ and $\xi_{f}$ decrease with increasing $V_{e}$ and finally vanish at the TCP given by

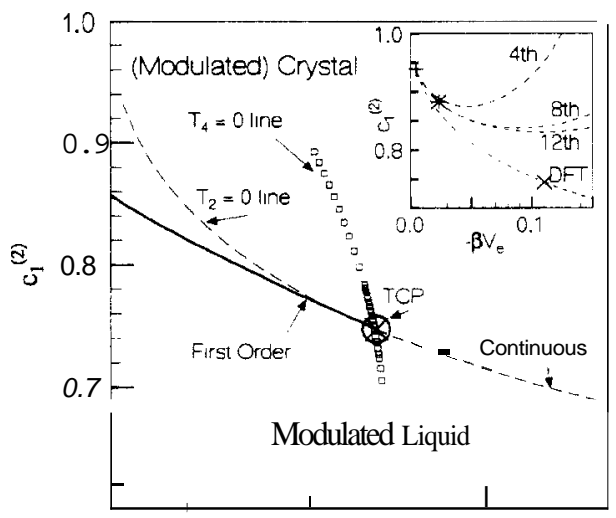

$-\beta V_{e}=0.106$ and $c_{1}^{(2)}=0.748$. Thereajter, one has a continuous transition from the modulated liquid to the (modulated) crystalline phase across the dashed line in Fig. 1. Illustrative behavior of $\xi_{i}$ and $\xi_{d}$ as $\left({ }^{(2)}\right.$ increases for two fixed values of $-\beta V_{e}(0.015$ and 0.15$)$ characterizing the two types of transition is shown in Figs. 2(a) and 2(b).

Qualitatively similar results arise in a similarly simplified DFT in 3D, if we take a $V_{e}$ which is 2 dimensional. Here, when $V_{e}=0$, the liquid freezes $[5,6]$ at $c_{1}^{(2)}=0.7\left(S_{\max }=3.3\right)$ into a bcc crystal with the 12 $\operatorname{RLV's}\left\{\vec{g}_{i}^{(o)}\right\}=( \pm 1, \pm 1,0) q_{0} / \sqrt{2},(0 . \pm 1 . \pm 1) q_{0} / \sqrt{2}$, and $( \pm 1,0 . \pm 1) q_{0} / \sqrt{2}$. When a $2 \mathrm{D}$ modulation $V_{e}$ characterized by the four wave vectors $\left\{\vec{g}_{i}^{(f)}\right\}=( \pm 1, \pm I .0) q_{0} / \sqrt{2}$ is turned on, once again the first-order transition from the modulated liquid to the (modulated) crystal changes into a continuous transition in a way similar to that shown in Fig. 1, at a TCP given by $-\beta V_{e}=0.22$ and $c_{1}^{(2)}=0.55$.

We can establish a general criterion which the wave vector set $\left\{\vec{g}_{i}^{(f)}\right\}$ characterizing $V_{e}$ and its complementary set $\left\{\vec{g}_{i}^{(d)}\right\}$ must satisfy, in order that the (modulated) liquid crystal transition changes from first order to a continuous one as $V_{e}$ increases. The criterion is as follows:

An odd combination of vectors of $\left\{\vec{g}_{i}^{(d)}\right\}$
$\quad \neq$ any integer combination of rhe vectors of $\left\{\vec{g}_{i}^{(f)}\right\}$.

For, if condition (2) is satisfied, it can be verified easily by expanding the $\ln \Phi$ term in (1) that the Landau expansion for $\beta F$ in powers of $\xi_{d}$, given $\xi_{f} \neq 0$ (with $\xi_{f}$ treated nonperturbatively), has only even powers of $\xi_{d}$; the coefficients, which we can compute numerically, are functions of $-\beta V_{e}$ and $c_{1}^{(2)}$. As $c_{1}^{(2)}$ and $-\beta V_{e}$ increase, the coefficient of the second-order term in this Landau expansion, $T_{2}$, changes sign and becomes negative, leading to an instability with respect to the formation of $\xi_{d}$. In the region of the parameter space where the fourth-order coefficient, $T_{4}$, is positive, this leads to a continuous transition. However, if there is also a region of parameter space where $T_{4}$ is negative (but $T_{6}$ is positive), the continuous transition is preempted by a first-order transition. The TCP thus arises when both $T_{2}$ and $T_{4}$

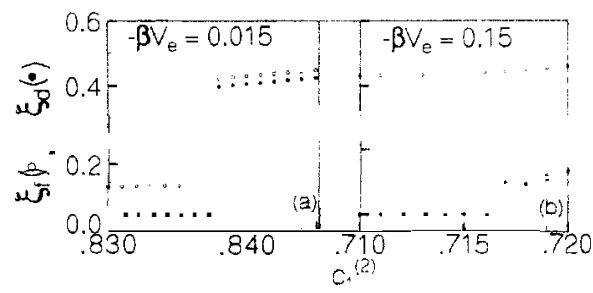

FIG. 2. $\xi_{f}$ and $\xi_{d}$ as functions of $c_{1}^{(2)}$ showing a first-order modulated liquid $\rightarrow$ crystal transition for $-\beta V_{t}=0.015$ (a) and a continuous transition for $-\beta V_{t}=0.15(b)$. 
become zero. In the 2D case, corresponding to Fig. 1, we also show in the figure the lines along which $T_{2}=0$ (marked by dashes) and $T_{4}=0$ (marked by small squares). To the left of the $T_{4}=0$ line, where $T_{4}$ is negative, the first-order transition (solid line) preempts the continuous transition. The $T_{4}=0$ line meets the $T_{2}=0$ line at the tricritical point. In this way we obtain the precise location of the tricritical points quoted earlier. It is easy to verify that the above condition is satisfied in the context of the modulations discussed above [13]. Note in particular that in the 3D bcc case, a $1 \mathrm{D}$ set or any arbitrary 2D set for $\left\{\vec{g}_{i}^{(f)}\right\}$ picked out from $\left\{\vec{g}_{i}^{(o)}\right\}$ would not satisfy (2).

It is interesting at this point to compare the above phase diagram with that obtainable in the Landau theory of Chowdhury and co-workers [1]. For this purpose we expanded the conventional Ramakrishnan-Yussouff $[5,6]$ density functional free energy in powers of the Fourier components of $\rho(\vec{r}) / \rho_{0}$ for the wave vectors $\left\{\vec{g}_{i}^{(f)}\right\}$ and $\left\{\vec{g}_{i}^{(d)}\right\}$ in the 2D context. We truncated the power series at different powers and found the phase diagram by minimizing the resulting free energy. We studied truncations up to the 12 th order with the results as shown in the inset of Fig. 1. In each of these cases we obtain a TCP as marked; however, the numbers are very different from, and converge very slowly to, those of the full density functional theory. More importantly, the continuous transition line eventually bends upwards for large enough $V_{e}$, indicating that there is no stable crystal phase for large $V_{e}$ in these truncated Landau theories, in contrast to the DFT result, where the critical line asymptotes to $c_{1}^{(2)}=0.5$ for large $-\beta V_{e}$.

The condition (2) is all important, for if it is satisfied, the existence of the $T C P$ is robust with respect to the inclusion of the effect of finite compressibility, of larger RLV shell order parameters, and of higher order correlations in the DFT. To see this we partition the (full) RLV set of the crystal into a set $\left\{K^{(f)}\right\}$, the reciprocal lattice of the modulated phase, which includes $\overrightarrow{0},\left\{\vec{g}_{i}^{(f)}\right\}$, and the set $\left\{\vec{G}_{\alpha}^{(f)}\right\}$ obtained by (new) integer combinations of $\left\{\vec{g}_{i}^{(f)}\right\}$; and the complimentary set $\left\{K^{(d)}\right\}$ which includes $\left\{\vec{g}_{i}^{(d)}\right\}$ and the remaining $\operatorname{RLV's}\left\{\vec{G}_{l}^{(d)}\right\}$. The order parameters for these five classes are denoted by $\xi_{0}, \xi_{f}, \xi_{\alpha}^{(f)}, \xi_{d}$, and $\xi_{l}^{(d)}$, respectively. For the large $c_{0}^{(2)}$ values relevant to our context, to $O\left(1 / c_{0}^{(2)}\right)$ one has $\xi_{0}=-\ln \Phi\left(1+1 / c_{0}^{(2)}\right)$ and the DFT free energy to be minimized is

$$
\begin{aligned}
\beta F= & -\ln \Phi+(\ln \Phi)^{2} / 2 c_{0}^{(2)}+\frac{1}{2 c_{1}^{(2)}}\left(n_{f} \xi_{f}^{2}+n_{d} \xi_{d}^{2}\right) \\
& +\sum_{\alpha} \frac{1}{2 c_{\alpha}^{(2)}}\left[\xi_{\alpha}^{(f)}\right]^{2}+\sum_{l} \frac{1}{2 c_{l}^{(2)}}\left[\xi_{l}^{(d)}\right]^{2}+\frac{n_{f} \xi_{f}}{c_{1}^{(2)}} \beta V_{e}
\end{aligned}
$$

where now $\Phi \equiv v_{0}^{-1} \int_{\vec{r}}^{\text {cell }} d^{d} \vec{r} \exp \left[\xi_{f} \phi_{f}(\vec{r})+\xi_{d} \phi_{d}(\vec{r})+\right.$ $\left.\sum_{\alpha} \xi_{\alpha}^{(f)} \exp \left(i \vec{G}_{\alpha}^{(f)} \cdot \vec{r}\right)+\sum_{l} \xi_{l}^{(d)} \exp \left(i \vec{G}_{l}^{(d)} \cdot \vec{r}\right)\right]$. In the modulated liquid phase $\xi_{d}=\xi_{l}^{(d)}=0$. With respect to this phase, consider the Landau expansion for the free energy in terms of $\xi_{d}$ and $\xi_{l}^{(d)}$ to quadratic order. This will now have cross terms, determined by $\int^{\text {cell }} \phi_{d} \exp \left(i \vec{G}_{l}^{(d)} \cdot \vec{r}\right) \exp \left[\xi_{f} \phi_{f}+\sum_{\alpha} \xi_{\alpha}^{(f)} \exp \left(i \vec{G}_{\alpha}^{(f)} \cdot \vec{r}\right)\right]$. Now only those $\xi_{l}^{(d)}$ for which such integrals are nonzero can affect the quadratic instability of $\xi_{d}$. This happens only if $\vec{G}_{l}^{(d)}$ is a linear combination of an arbitrary number of vectors from the set $\left\{\vec{g}_{i}^{(f)}\right\}$ and one from the set $\left\{\vec{g}_{i}^{(d)}\right\}$. Let us write the resulting quadratic form as $T_{d d}^{(2)} \xi_{d}^{2}+2 T_{d l}^{(2)} \xi_{(d)} \xi_{l}^{(d)}+T_{l m}^{(2)} \xi_{l}^{(d)} \xi_{m}^{(d)}$. Now the dominant instability of the modulated liquid phase will be determined by the order parameter mode $\tilde{\xi}_{d}$ which is that particular linear combination of $\xi_{d}$ and $\xi_{l}^{(d)}$ (typically, mostly $\xi_{d}$ plus a little bit of other $\xi_{l}^{(d)}$ ) which corresponds to the lowest eigenvalue of this (Hessian) matrix $\mathbf{T}^{(2)}$. The instability sets in when this lowest eigenvalue of $\mathbf{T}^{(2)}$ crosses zero and goes negative. The key point is that if condition (2) is satisfied, general symmetry reasons [14] ensure that the Landau expansion does not contain any odd powers of $\tilde{\xi}_{d}$ either. So the TCP in the phase diagram will be retained, but relocated, being now given by the condition of simultaneous vanishing of the cofficients of $\left(\tilde{\xi}_{d}\right)^{2}$ and $\left(\tilde{\xi}_{d}\right)^{4}$ in the Landau expansion. The effect of finite compressibility, which arises when $c_{0}^{(2)} \equiv \rho_{0} c^{(2)}(q=0) \neq-\infty$, is taken care of by $\xi_{0}$ which does not affect the quadratic instability of $\xi_{d}$. Hence the tricritical point is robust under the effect of finite compressibility, too. We have explicitly verified these conclusions by numerical calculations including the effects of the order parameters corresponding to wave vectors $\left\{\vec{g}_{2 i}\right\}$ near the second peak in $c^{(2)}(q)$ and $\xi_{0}$. The resulting relocated TCP's are listed in Table I. When $c_{0}^{(2)} \neq-\infty$, there is a change in density at the firstorder liquid solid transition, but this decreases with increasing $V_{e}$ and vanishes at the TCP.

Finally, we discuss our DFT calculation of laser induced freezing in real colloidal systems [8]. For this purpose we considered the same experimental system as Monovoukas and Gast [15] for which the DFT phase diagram is known in the absence of $V_{e}$ [7]. The liquid state DCF for this system of charged colloidal particles with diameter $1334 \AA$ and surface charge $880 e$ was obtained from the rescaled mean spherical approximation of Hansen and Hayter [7,8,16] using the model Derjaguin-Landau-Verweg-Overveek potential [8]. We focused our attention on the portion of the phase diagram

TABLE I. TCP coordinates for various versions of DFT.

\begin{tabular}{ccccccc}
\hline $\begin{array}{c}\text { Crystal lattice } \\
\text { when } V_{e}=0\end{array}$ & \multicolumn{3}{c}{ 2D triangular } & \multicolumn{3}{c}{$3 \mathrm{D}$ bcc } \\
\hline $\begin{array}{c}\left\{( \pm 1, \pm \sqrt{3}) q_{0}\right. \\
\left.\vec{g}_{2 i}\right\}\end{array} c^{(2)}\left(g_{2 i}\right)$ & 0 & 0 & 0.16 & 0 & 0 & 0.07 \\
$c_{2}^{(2)}$ & $-\infty$ & -51.5 & $-\infty$ & $-\infty$ & -40 & $-\infty$ \\
$\mathrm{TCP}:-\beta V_{e}$ & 0.106 & 0.114 & 0.117 & 0.219 & 0.237 & 0.228 \\
$c_{1}^{(2)}$ & 0.748 & 0.741 & 0.731 & 0.520 & 0.507 & 0.508 \\
\hline \hline
\end{tabular}


where there is a first-order transition from the liquid to a bcc phase in the absence of $V_{e}$. We took the modulation wave vectors $\left\{\vec{g}_{i}^{(f)}\right\}$ of the external potential to be along $( \pm 1, \pm 1,0) q_{0} / \sqrt{2}$ as before. We did calculations retaining order parameters corresponding to 10,20 , and 50 shells of the RLV's of the bcc lattice. We retained the three-body terms in the DFT [5-7] in the same spirit as in Ref. [7] with $c^{(3)}=0.23$. In the 3D parameter space of impurity concentration $n_{i}$, volume fraction $\phi$, and $-\beta V_{e}$, the first-order and continuous transitions between the modulated liquid and the crystal now take place across surfaces, which meet in a line of TCP. We find that in the $n_{i}$ ranges $(1.8-2.6) \times 10^{-9} \mathrm{~mole} / \mathrm{cm}^{3},-\beta V_{e}$ is almost a constant $\simeq 0.198$ along the tricritical line (compared with $-\beta V_{e}=0.22$ obtained in the simplest theory). The corresponding electric field strength in the laser beam is $E_{c} \simeq 8.0 \times 10^{4} \mathrm{~V} / \mathrm{m}^{3}[17]$. So in the above range of $\phi$ and $n_{i}$ values, this system should show first-order and continuous liquid solid transitions for $E<E_{\varepsilon}$ and $E>E$, respectively.

In conclusion, we have presented in this paper the results of a DFT for laser-induced freezing in colloidal systems which definitely predicts, for appropriately chosen modulation potentials, the existence of tricritical points and critical lines in the transition from the modulated liquid to the (modulated) crystal for large laser power. Such predictions are also borne out by our own recent Monte Carlo simulations [18]. We hope to report in the future on many other fascinating questions that can be asked about these critical points, namely their robustness with respect to the inclusion of fluctuation effects (especially in the $2 \mathrm{D}$ context), their light scattering characteristics, their universality class, etc. Meanwhile, our results suggest that experimental studies of colloidal systems with a view to investigating these issues would be of great interest. We may mention that the DFT presented here for laser induced freezing is rather general and should be applicable with some modifications to other situations of field induced ordering such as in ferrofluids, in electrorheological fluids, and in adsorbates on substrates (where the ordering is induced by the substrate potential).

We thank the Council for Scientific and Industrial Research and the Indo-French Centre for the Promotion of Advanced Research for financial support; the Supercomputer Education and Research Centre at the Indian Institute of Science for computing facilities; and Sriram Ramaswamy, T.V. Ramakrishnan, Surajit Sengupta, Siddhartha Shankar Ghosh, Gautam I. Menon, and Pinaki Majumdar for discussions.

*Also at Jawaharlal Nehru Center for Advanced Scientific Research, IISc Campus, Bangalore 560 012, India.

[1] A. Chowdhury, B.J. Ackerson, and N.A. Clark, Phys. Rev. Lett. 60, 833 (1985); also B. J. Ackerson and A. H. Chowdhury, Faraday Discuss. Chem. Soc. 83, 309 (1987).

[2] L. Landau and E.M. Lifshitz, Statistical Physics, Course on Theoretical Physics, Vol. 5 (Pergamon Press,
New York, 1985), Part I, 3rd ed.; S. Alexander and J. McTague, Phys. Rev. Lett. 41, 702 (1978).

[3] K. Loudiyi and B.J. Ackerson, Physica (Amsterdam) 184A, 1 (1992).

[4] K. Loudiyi and B. J. Ackerson. Physica (Amsterdam) 184A, 26 (1992).

[5] T.V. Ramakrishnan and M. Yussouff, Phys. Rev. B 19, 1775 (1979); T.V. Ramakrishnan. Pramana 22, 365 (1984).

[6] For a recent review. see Y. Singh, Phys. Rep. 207. 35 I (1991).

17] S. Sengupta and A.K. Sood. Phys. Rev. A 44. 1233 (1991).

[8] A.K. Sood, in Solid State Physics, edited by E. Ehrenreich and D. Turnbull (Academic Press. New York, 1991). Vol. 45. p. 1

[9] H. Xu and M. Baus, Phys. Lett. A 117. 127 (1986); see also J.L. Barrat and H. Xu. J. Phys. Condens. Matter 2. 9445 (1990).

[10] T. V. Ramakrishnan, Phys. Rev. Lett. 48, 541 (1982).

[11] H. R. Krishnamurthy (unpublished); S. Sengupta, thesis, Indian Institute of Science. Bangalore. India, 1991 (unpublished).

[12] We note that in contrast to the Landau-type [2] freeenergy functions, the free energy (1) is a nonpolynomial function, with arbitrary powers in a Landau expansion being present. Furthermore, the coefficients of the expansion are uniquely fixed given $c_{1}^{(2)}$ and $-\beta V_{\epsilon}$.

[13] Thus we disagree with the conclusions of [9] that the transition is always first order except in the case where $V_{e}$ has the full symmetry of the crystal (for which, trivially, there is no phase transition). The reason for the discrepancy is that the wave vectors of the modulations chosen in [9] do not satisfy our condition (2) in the context of the liquid to fcc transitions studied in these papers. The simplest $\left\{\vec{g}_{l}^{(f)}\right\}$ for the fcc case for which the symmetry condition (2) can be satisfied is the choice $(1,1,1) \frac{q 0}{\sqrt{3}},(-1,-1,-1) \frac{90}{\sqrt{3}},(1,1,-1) \frac{90}{\sqrt{3}}$, and $(-1,-1,1) \frac{90}{\sqrt{3}}$.

[14] A general term in the Taylor expansion for the total excess free energy obtained from (3) about the modulated liquid phase has the form

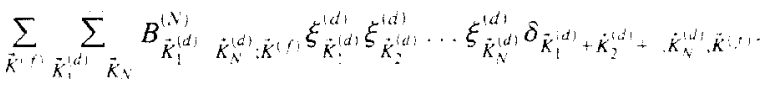
Because of condition (2) an odd number of-vectors from $\left\{K^{(d)}\right\}$ can never be equal any member of $\left\{\dot{K}^{(f)}\right\}$, and the $\delta$ function restriction above cannot be satisfied for any odd $N$.

[15] Y. Monovoukas and A.P. Gast. J. Colloid Interface Sci. 128. 533 (1989).

[ 16J J. P. Hansen and J. B. Hayter, Mol. Phys. 25, 651 (1982).

[17] Our estimate of the laser electric field $E$ has been made using $V_{e}=\frac{1}{2} \chi E^{2}$, where $\chi$ is the dielectric susceptibility of the colloidal particles. $\chi$ has been estimated as $\frac{1}{2} \frac{n_{1}^{2}-n_{2}^{2}}{\left(n_{1} / n_{2}\right)^{2}+2} a^{3}$ [see M.M. Burns, J. M. Fournier, and J.A. Golovchenko, Science 249, 749 (1990)], where $n_{1}$ and $n_{2}$ are, respectively, the refractive indices of the colloidal particles and the solvent, and $\boldsymbol{a}$ is the radius of the particles.

[18] J. Chakrabarti. S. Sengupta, H.R. Krishnamurthy, and A. K. Sood (to be published). 